\title{
EDITORIAL
}

\section{Tuberculosis drug resistance in Europe: sunny days, but clouds on the horizon?}

\author{
N.W. Schluger
}

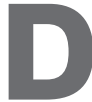

rug-resistant tuberculosis (TB) has now emerged as a public health concern of major proportions. Although drug resistance was recognised in the British Medical Research Council's landmark streptomycin trial [1], there was little evidence that widespread resistance to multiple anti-TB drugs was a significant public health concern until New York City (NY, USA) reported that nearly 12\% of all its cases in 1992 were resistant to at least isoniazid (INH) and rifampicin [2]. Such cases were termed multidrug resistant (MDR). Many of these cases occurred in persons infected with HIV, and there was evidence that many of the infected patients acquired the infection in hospitals or other congregate settings, including prisons [3]. Mortality associated with these cases was extremely high [4].

In 1998, the World Health Organization (WHO) published the results of a global survey of drug-resistant TB, conducted from 1994-1997 [5]. Overall, among patients with no previous treatment for $\mathrm{TB}, \mathrm{INH}$ resistance had a prevalence of $7.3 \%$, and the overall prevalence of MDR-TB was $1.4 \%$. Among previously treated patients, the prevalence of multidrug resistance was $13 \%$. Resistance was present in all regions of the globe, although there were particular concentrations of cases in Russia and the countries of the former Soviet Union, Asia, the Dominican Republic and Argentina. In some of these countries, MDR-TB rates exceeded $10 \%$, even in previously untreated patients.

The response to the emergence of MDR-TB was mostly focused on using the DOTS (directly observed therapy, short-course) strategy, largely as a means of preventing the emergence of new cases of MDR-TB. The DOTS strategy, the key component of WHO's global TB control efforts, seeks to assure that, in addition to the provision of medication to patients under direct observation, there is a steady and reliable availability of at least first-line drugs to all patients. This strategy seemed to achieve success in varied locations $[6,7]$.

Despite the success in some locations in reducing rates of drugresistant $\mathrm{TB}$, evidence has accumulated in the past several years that this problem is increasing in magnitude rather than decreasing. Epidemiologists from WHO estimate that the current global prevalence of MDR-TB is $\sim 425,000$ cases,

\section{STATEMENT OF INTEREST: None declared.}

CORRESPONDENCE: N.W. Schluger, Columbia University Medical Center, PH-8 East Room 101 622 West 168th Street, New York, NY 10032, USA. Fax: 1 2123425272. E-mail ns311@columbia.edu representing $4.3 \%$ of all new and previously treated cases [8]. This represents a significant increase from 2000, when it was estimated that there were $\sim 273,000$ cases. Of the cases of MDR$\mathrm{TB},>60 \%$ occur in China, India and the Russian Federation, but countries such as South Korea have substantial and increasing numbers of cases as well [9]. Most recently, of course, there has been renewed worldwide attention to the problem of drug resistance with the report of extensively drugresistant TB in patients with HIV infection in the KwaZuluNatal region of South Africa [10].

In response to the growing problem of MDR-TB, WHO has issued guidelines and recommendations. In describing a framework for effective control of drug-resistant TB, WHO recommends placing control efforts into the context of the DOTS strategy, the components of which are: a sustained political commitment; a rational case-finding strategy including accurate, timely diagnosis through quality-assured culture and drug susceptibility testing; appropriate treatment strategies that use second-line drugs under proper case management conditions; an uninterrupted supply of quality-assured anti-TB drugs; and a standardised recording and reporting system. The WHO guidelines specifically state that: "Surveillance of TB antimicrobial resistance is essential for providing information on the magnitude and trends in resistance, for developing treatment guidelines, and for monitoring the effect of interventions." Prior guidelines on surveillance in European countries underscored the importance of representative sampling, and collecting data on prior treatment and country of origin of patients, in order to identify high-risk persons and populations [11].

It is in this context that the report from KHUÊ et al. [12] should be viewed. They describe a national sentinel network of TB laboratories in France, a wealthy country with a low incidence of TB: 13 per 100,000 population in 2005, according to WHO. In 1995, a laboratory network based at university hospitals was created for TB surveillance. This network now includes 33 hospital laboratories with diverse geographical distribution, and standardised definitions of resistance were created for use in reporting from all labs. Although not linked to the national mandatory reporting system, an early report indicated that patients whose isolates were tested by the network appear to be representative of French TB patients [13]. The current paper presents data on 10 yrs of surveillance. The proportion of all French patients with TB captured in this network ranged from $10 \%$ in the early years to $29 \%$ more recently. Of TB patients in the Paris metropolitan area, $43 \%$ were covered by the network. 
In their paper covering 10 yrs of surveillance, KHUÊ et al. [12] present an interesting picture of the TB situation in France, which appears to be similar to that of many or most of the established market economy countries, which have relatively low TB incidence rates. Of the 13,283 patients captured by the network, $\geqslant 44 \%$ were born outside of France. The proportion increased from $39 \%$ at the establishment of the network to $53 \%$ in the most recent period, with most patients coming from subSaharan Africa, the Maghreb region (North and Northwest Africa) and Asia. HIV co-infection was present in $10 \%$ of patients. Roughly half of all cases were smear positive.

Of greatest interest is the information regarding drug resistance. Among persons never previously treated, resistance to any single drug was seen in $8.8 \%$ of cases, with streptomycin resistance $(6.6 \%)$ more common than $\mathrm{INH}$ resistance $(4.2 \%)$. As has been seen elsewhere [14], rifampicin monoresistance was more common in HIV-infected patients. There was no trend of increasing resistance in the period studied, though HIV infection, younger age and birth outside of France were associated with resistance in multivariate analysis. Among previously treated cases, resistance to at least one drug was $20.6 \%$, with similar risk factors as for untreated patients.

Overall rates of MDR-TB were low. Among previously untreated patients, INH and rifampicin resistance was seen in $0.7 \%$, though among HIV-infected persons born outside of France, the rate was $2.7 \%$. Of previously treated patients, $6.9 \%$ had resistance to INH and rifampicin, again with increased rates among those born outside France.

What are the important messages from this study? In many ways, France is typical of most resource-rich countries: the overall rate of TB is low; a majority, or near majority, of cases occur in persons who have immigrated recently; and multidrug resistance in general is uncommon, although rates are higher in recently arrived persons, previously treated individuals and HIV-infected patients. These findings demonstrate the value of the French surveillance network and provide important guidance to providers caring for TB patients in that country, regarding both initial empirical therapy for $\mathrm{TB}$ and treatment for patients who have had prior episodes of TB. In the current global context of TB epidemiology, the value of such a network is obvious and it underscores the need for greatly enhanced surveillance, based on culture and drug susceptibility testing in high-burden countries and those countries with higher rates of MDR-TB. Although the cost of such expanded surveillance is high, the cost of not having this kind of surveillance is even higher.

Though reassuring, it still seems somewhat surprising that the rates of drug resistance reported in the study by KHUÊ et al. [12] are as low as they are. Is there any reason to think that they might in fact be higher? The current report ends with data collected in 2004. The last 3 yrs have seen the development and spread of extensively drug resistant (XDR)-TB and globally there is no indication that overall MDR rates are decreasing. Migration of populations from East to West and North to South continues, and may be accelerating. As MigLIORI et al. [15] pointed out, of 21 countries in which at least one case of XDRTB has been reported, 10 border Europe. For these reasons, the current report should be a spur for increased vigilance and surveillance, and does not provide any justification for complacency.

What are the limitations of the study? The major limitation is that the surveillance network is not linked directly to the mandatory notification system and is thus not comprehensive. However, it does seem as though cases in the network are highly representative of all cases of TB in France. Still, one is left to wonder if cases not reported to this network are in fact more likely to represent those units within the French system that are functioning poorly, and which might have a higher incidence of MDR-TB.

Overall, the report by KHUÊ et al. [12] provides an excellent demonstration of the value of country-wide surveillance and drug susceptibility testing. The data are valuable and generally reassuring, although they point to the need for tailored treatment of persons whose demographic characteristics put them at high risk for disease with drug-resistant strains of tuberculosis.

\section{REFERENCES}

1 Medical Research Council Streptomycin in Tuberculosis Trials Committee. Streptomycin treatment for pulmonary tuberculosis. Br Med J 1948; 2: 769-782.

2 Frieden TR, Sterling T, Pablos-Mendez A, Kilburn JO, Cauthen GM, Dooley SW. The emergence of drug-resistant tuberculosis in New York City. N Engl J Med 1993; 328: 521-526.

3 Frieden TR, Sherman LF, Maw KL, et al. A multiinstitutional outbreak of highly drug-resistant tuberculosis: epidemiology and clinical outcomes. JAMA 1996; 276: 1229-1235.

4 Park MM, Davis AL, Schluger NW, Cohen H, Rom WN. Outcome of MDR-TB patients, 1983-1993. Prolonged survival with appropriate therapy. Am J Respir Crit Care Med 1996; 153: 317-324.

5 Pablos-Mendez A, Raviglione MC, Laszlo A, et al. Global surveillance for antituberculosis-drug resistance, 19941997. World Health Organization-International Union against Tuberculosis and Lung Disease Working Group on Anti-Tuberculosis Drug Resistance Surveillance. N Engl J Med 1998; 338: 1641-1649.

6 Frieden TR, Fujiwara PI, Washko RM, Hamburg MA. Tuberculosis in New York City-turning the tide. N Engl J Med 1995; 333: 229-233.

7 Weis SE, Slocum PC, Blais FX, et al. The effect of directly observed therapy on the rates of drug resistance and relapse in tuberculosis. N Engl J Med 1994; 330: 1179-1184.

8 Zignol M, Hosseini MS, Wright A, et al. Global incidence of multidrug-resistant tuberculosis. J Infect Dis 2006; 194: 479-485.

9 Bai GH, Park YK, Choi YW, et al. Trend of anti-tuberculosis drug resistance in Korea, 1994-2004. Int J Tuberc Lung Dis 2007; 11: 571-576.

10 Gandhi NR, Moll A, Sturm AW, et al. Extensively drugresistant tuberculosis as a cause of death in patients coinfected with tuberculosis and HIV in a rural area of South Africa. Lancet 2006; 368: 1575-1580.

11 Schwoebel V, Lambregts-van Weezenbeek CS, Moro ML, et al. Standardization of antituberculosis drug resistance 
surveillance in Europe. Recommendations of a World Health Organization (WHO) and International Union Against Tuberculosis and Lung Disease (IUATLD) Working Group. Eur Respir J 2000; 16: 364-371.

12 Khuê PM, Truffot-Pernot C, Texier-Maugein J, Jarlier V, Robert J. A 10-year prospective surveillance of Mycobacterium tuberculosis drug resistance in France 19952004. Eur Respir J 2007; 30: 937-944.

13 Robert J, Trystram D, Truffot-Pernot C, Carbonnelle B, Grosset J. Surveillance of Mycobacterium tuberculosis drug resistance in France, 1995-1997. AZAY Mycobacteria Study Group. Int J Tuberc Lung Dis 2000; 4: 665-672.

14 Sandman L, Schluger NW, Davidow AL, Bonk S. Risk factors for rifampin-monoresistant tuberculosis: a casecontrol study. Am J Respir Crit Care Med 1999; 159: 468-472.

15 Migliori GB, Loddenkemper R, Blasi F, Raviglione MC. 125 years after Robert Koch's discovery of the tubercle bacillus: the new XDR-TB threat. Is "science" enough to tackle the epidemic? Eur Respir J 2007; 29: 423-427. 